[10] Sanders. J. of Dairy Sc, 1938, 21, 153 et 1939, 22, 841.

[11] Sarran. Le Lait, 1932, 12, 789 .

[12] Shann, Hansen et Nutting. J. oj Dairy Sc., 1937, 20, 199.

[13] Sharp et Strubble. J. of Dairy Sc., 1935, 18, 527.

[14] Sjollema et Markus. Handl. v. b. Genv. t. Bevord. v. Melkk, 1923, 2,

I. analysé dans Le Lait, 1925, 5, 737.

[15] Tapernoux. J. Med. Vét., 1931, 83, 5.

[16] Tapernoux Rev. Vét., 1935, 86, 600.

[17] VolHard. J. prakt. Chem., 1874, 9, 217, et Ann. d. Chem., 1878, 190, 1.

\title{
ANALYSE DU LAIT PAR LES MÉTHODES PHOTO-COLORIMETRIQUES
}

par

\section{Docteur Ingénieur J.-L. de HAUSS}

La colorimétrie permet souvent le dosage de substances présentes en quantités minimes, difficilement décelables par les procédés de l'analyse classique volumétrique ou gravimétrique, d'où découlent les innombrables applications dans l'industrie chimique, la métallurgie, la biologie, etc. Le principe de ces méthodes colorimétriques réside dans la mesure de l'intensité de la couleur produite par une réaction chimique où intervient le corps recherché.

Les anciens colorimètres procédaient par enfoncement plus ou moins grand dans deux cures de deux lames à faces parallèles en verre. La différence de hauteur nécessaire pour donner un éclairement égal sur deux plages visibles dans un oculaire permettait de déterminer la transparence, done la quantité de produits à doser.

Tous ces appareils d'optique de précision ne permettaient que des mesures visuelles; les variations visuelles et les indications obtenues dépendaient des qualités particulières de la vision de l'observateur et par conséquent les erreurs d'appréciation des solutions colorées à l'œil pouvaient donner des grands écarts souvent inadmissibles au point de vue analytique.

L'adaptation de la photo-électricité à la colorimétrie a apporté un perfectionnement permettant de réduire toutes les erreurs au minimum par le fait même que les yeux de l'observateur ne servent plus à comparer l'intensité de la coloration, mais interviennent uniquement pour la lecture des courants produits par la cellule photo-électrique. Les mesures sont à la fois précises et rapides.

La cellule photo-électrique mesure tout phénomène susceptible de se manifester par un effet lumineux. Sa sensibilité varie suivant la lumière incidente, e'est-à-dire, la valeur du courant photo- 
électrique produit par l'élément photo-sensible dépend à la fois de la qualité de la lumière et de son intensité.

Ces comparaisons sont donc possibles à condition de décomposer la mesure globale en une série de mesures en lumière pratiquement monochromatique. La décomposition de la lumière blanche (celle d'une lampe à incandescence) peut être faite au moyen d'un jeu, par exemple de 6 filtres colorés correspondant aux six couleurs fondamentales du spectre solaire : violet, bleu, vert, jaune, orangé et rouge. Ces filtres peuvent être choisis suivant les exigences de l'analyse, de façon à donner une bande de longueurs d'onde assez étroite permettant d'obtenir une lumière monochromatique bien sélective.

La précision des mesures dépend avant tout du soin apporté dans la présentation des échantillons à comparer ou à examiner.

Le courant photo-électrique est mesuré directement à l'aide du microampèremètre ou d'un galvanomètre à miroir. Ce courant est proportionnel à l'intensité de la lumière reçue par l'élément photosensible.

Un grand avantage de l'emploi de la photo-colorimétrie est surtout celui que les mesures ne dépendent plus, de la sensibilité et de la défectuosité de l'œil humain.

La sensibilité de l'œil varie, comme on le sait, en fonction de la longueur d'onde : elle est nulle aux deux extrémités du spectre à 4.000 et 7.000 angströms et son maximum se place aux environs de 5.500 angströms dans la bande verte. La défectuosité de la vision humaine jouait d'ailleurs dans les mesures colorimétriques un rôle d'erreurs importantes. Ces erreurs étaient dues soit à la trichromaticité incomplète de l'œil de l'observateur qui se manifestait par manque de sensibilité pour les différentes couleurs, soit à l'effet connu sous le nom de phénomène de Purkyne caractérisé par le déplacement vers les petites longueurs d'onde de la courbe de visibilité relative en fonction de la lumière. Le maximum de sensibilité qui se place aux environs de 5.500 angströms pour l'œil moyen en lumière diurne, a sa valeur de 5.000 angströms en lumière crépusculaire.

Un colorimètre photo-électrique muni d'une cellule photosensible au sélénium ayant subi un vieillissement préalable de dix mois, en vue d'assurer sa fidélité et sa stabilité, convient le mieux pour cette sorte de mesure.

L'appareil peut être alimenté par le secteur de 110 à 220 volts (courant alternatif de 50 cycles) ou par un accumulateur de 6 volts.

La lampe à incandescence de très faible intensité est pratiquement froide et n'altère en aucune façon les liquides examinés. Elle constitue un facteur de stabilité pour la cellule photo-électrique 
et permet d'examiner toutes les substances pouvant subir un changement de composition chimique sous l'influence des variations de température.

De plus, étant donné que le colorimètre est muni d'un régulateur automatique de tension, la tension aux bornes de la lampe est maintenue rigoureusement constante malgré les variations du secteur allant jusqu'à $15 \%$.

En résumé, le principe de ces méthodes est basé sur la mesure du courant produit par la cellule photo-électrique sous l'influence d'un flux lumineux d'intensité connue traversant le liquide examiné. Le colorimètre photo-électrique utilisant la méthode par déviation donne la concentration en substance recherchée grâce. à une courbe de référence établie une fois pour toutes.

En ce qui concerne la précision dans l'appréciation visuelle de la luminosité d'une substance, on arrive à une précision de $\pm 1 \%$. Ceci ne vaut encore que dans la région jaune-vert du spectre dans laquelle l'œil humain a sa plus grande acuité. Dans les régions bleues et rouges du spectre, physiologiquement moins favorables, la précision de l'acuité visuelle faiblit sensiblement. Les colorimètres photo-électriques donnent une précision de quelques dixièmes $\%$ et cette précision ne se limite pas à une région déterminée du spectre. Les solutions de couleur rouge ou bleue sont également favorables à la colorimétrie photo-électrique avec les éléments photo-sensibles au sélénium.

Grâce à cette nouvelle conception de la colorimétrie, on a pu mettre au point une série de méthodes d'analyses permettant de doser presque toutes les substances de l'organisme humain. Mais en dehors de ces recherches, la colorimétrie photo-électrique a trouvé un grand champ d'application dans toutes les activités industrielles. C'est ainsi que les exemples d'utilisation des mesures colorimétriques en chimie alimentaire se multiplient continuellement. On dose les protéines, l'amidon, le sucre, l'albumine ainsi que les métaux lourds tels que le plomb, le cuivre, le fer dans les denrées alimentaires. Les dosages de l'acide nicotinique et de la nicotinamide, ainsi que les dosages des vitamines se font actuellement par la colorimétrie photo-électrique d'une façon courante.

Les dosages des matières grasses du lait se font à l'aide de turbidimètres dans lesquels on observe la disparition d'un index de couleur foncée, soit à l'aide de colorimètres dans lesquels en plongeant une tige de verre bleu, on provoque un reflet bleuâtre dont l'intensité est inversement proportionnelle à la teneur en graisse. Tous ces appareils primitifs sont progressivement supplantés par les colorimètres photo-électriques donnant des résultats objectifs et exacts. 
Les applications de la colorimétrie photo-électrique dans l'industrie laitière deviennent de plus en plus nombreuses et éliminent les anciennes méthodes peu précises et exigeant un temps proportionnellement trop long.

Tel est, par exemple, le dosage photo-colorimétrique du calcium dont la teneur dans le lait est variable suivant l'époque et le terrain de pâturage.

L'analyse des eaux et le traitement des eaux résiduaires se font également très couramment par ces méthodes caractérisées par un gain considérable de temps et un emploi de très petites quantités de produits.

Signalons que les mesures de $p$ H ont reçu des applications aussi nombreuses que diverses, surtout pour l'examen des eaux. Nous donnons un exemple d'emploi du para-nitrophénol comme indicateur de $p$ H dans la zone de 5 à 7,4 .

L'étalonnage de l'appareil se fait au moyen de solutions tampons suivant MaC Ilvaine (Analyse Colorimétrique par B. Lange, page 506, édition Dunod, Paris, 1947). Ces solutions se préparent très facilement au laboratoire.

L'indicateur est constitué par une solution hydro-alcoolique de para-nitrophénol N/100.

La quantité d'indicateur requise est de $0,6 \mathrm{~cm}^{3}$ pour très exactement $10 \mathrm{~cm}^{3}$ de liquide examiné.

Le témoin de référence à porter à la lecture 100 est constitué par l'eau distillée. Le filtre employé est le filtre violet.

Nous donnons l'allure de la courbe de $p H$ ainsi relevée, représentée par la figure suivante :

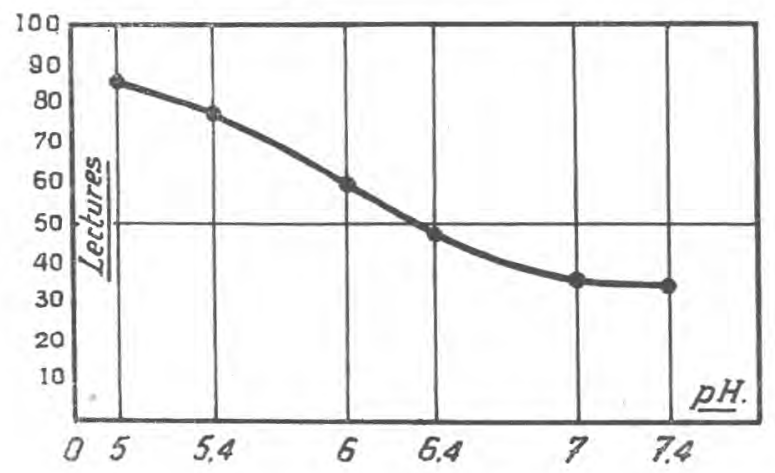

Une simple lecture de la déviation du microampèremètre permet de mesurer la valeur du $p \mathbf{H}$ cherché.

On constate que les plages de lectures sont larges et permettent d'apprécier facilement le dixième de $p \mathrm{H}$. 
Ces mesures ont été faites par notre collègue $D^{r}$ J.-E. GoRIs, avec le colorimètre Electro-Synthèse, modèle Industriel équipé de 6 filtres monochromatiques dont les longueurs d'onde sont les suivantes :

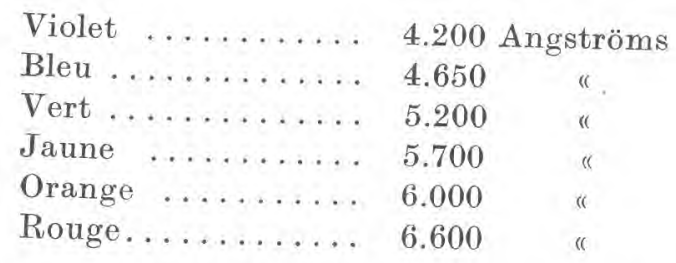

Pour déterminer ce bref exposé, soulignons simplement que toutes les anciennes méthodes colorimétriques ont été facilement adaptées à la colorimétrie photo-électrique qui, à son tour, a augmenté sensiblement la rapidité et la précision des mesures.

\section{EXTRAIT BIBLIOGRAPHIQUE}

SNELl and SNELL. Colorimetric methods of analysis. D. Van Nostrand Company Ine., 1947.

A. Thise. Absolutkolorimetrie. Walter de Gruyter und Co, Berlin, 1939. Kontum Gustav. Kolorimetrie und Spektralphotometrie. Lange, Maxwell
et Spxinger, édition Paris, Londres. P. Fleury. Couleurs et colorimétrie. Librairie Scientifique Hermann et Co,
Paris.

Ch. Fabry. La colorimétrie, introduction générale à la photométrie. Edition de la Revue Optique, Paris.

F. Blottiau. Colorimétrie. Edition de la Revue Optique, Paris.

P. J. Bouma. Les couleurs et leur perception visuelle. Edition Philips, dépositaire: Dunod, Paris.

J. L. DE HAUss. Les applications de la colorimétrie photo-électrique dans la chimie des eaux. L'Eau, no 12, décembre 1952.

\section{BIBLIOGRAPHIE ANALYTIQUE}

\section{LES LIVRES}

Rosell (J. M.) et Dos Santos (I.). - Metodos analiticos de laboratorio lactologico y microbiologia de las industrias lacteas. 2 vol. reliés, 913 et 663 pages, $18 \times 25$, très nombreux schémas, figures et photographies dont plusieurs en couleurs. Editeur : Labor S. A., Calle de Provenza, 88, Barcelona (Espagne) 1952. Prix : 30,60 dollars U.S.A.

L'origine de cet ouvrage considérable réside dans les années d'enseignement et de recherches des A., depuis 1929 pour le Pr Rosell au Collège de 